\title{
Comparación de la ingesta de energía y nutrientes en adolescentes mujeres con sobrepeso y obesidad
}

\author{
Jaime Pajuelo $\mathrm{R}^{1,2,3}$, Ivonne Bernui $\mathrm{L}^{1,4}$, Alicia Castillo $\mathrm{S}^{5}$, Sandra Cabrera $\mathrm{B}^{5}$, \\ Jhon Cuba $\mathrm{J}^{5}$ \\ ${ }^{1}$ Profesor Principal, Facultad de Medicina, Universidad Nacional Mayor de San Marcos. \\ ${ }^{2}$ Miembro Permanente, Instituto de Investigaciones Clínicas, Facultad de Medicina, UNMSM. \\ ${ }^{3}$ Médico Asistente, Servicio de Endocrinologia, Hospital Nacional Dos de Mayo. \\ ${ }^{4}$ Centro de Investigación en Bioquímica y Nutrición Facultad de Medicina, UNMSM. \\ ${ }^{5}$ Licenciado en Nutrición, Escuela Académico Profesional de Nutrición, UNMSM.
}

\begin{abstract}
Resumen
Introducción: El sobrepeso y la obesidad son consecuencia de una modificación de los patrones dietarios y de una actitud cada vez más sedentaria. Objetivos: Comparar la ingesta de energía y nutrientes de adolescentes mujeres con sobrepeso y obesidad. Diseño: Estudio transversal, observacional y analítico. Lugar: Centro Educativo de la zona urbana de Lima. Participantes: Adolescentes entre 10 a 18 años. Intervención: Se estudió 100 adolescentes con sobrepeso y 87 con obesidad. El diagnóstico se hizo mediante el indice de masa corporal (IMC), con la población de referencia de la Organización Mundial de la Salud (OMS) y con los niveles de corte entre 85 y 95 percentil para sobrepeso y más de 95 para obesidad. La evaluación de la cantidad de energía y de nutrientes se realizó mediante un cuestionario de frecuencia semicuantitativo, utilizando la Tabla Peruana de Composición de Alimentos y la de Departamento de Agricultura de los EE UU. Para el porcentaje de adecuación de la energía y para la identificación cuantitativa de la ingesta de vitaminas y mineral se utilizó las recomendaciones de la FAO. El análisis estadístico se hizo con la prueba t-Student para comparación de medias. Principales medidas de resultados: Energía ingerida, adecuación de los nutrientes, cantidad de vitaminas y minerales ingeridos, de acuerdo a los grupos estudiados. Resultados: No hubo diferencia de la ingesta de energía entre los grupos estudiados, y estos valores fueron menores que los requerimientos. La adecuación porcentual mostró que el consumo de las grasas estuvo por debajo del requerimiento; lo mismo sucedió con las grasas saturadas, monoinsaturadas y poliinsaturadas; sin embargo, lo que más se consumió fue la grasa saturada. En cuanto a las vitaminas y minerales, fueron ingeridas en cantidades que cubrían los requerimientos, a excepción de los folatos. La ingesta de fibra fue muy pobre. Conclusiones: Se puede afirmar que el consumo de energía en ambos grupos fue por debajo de su requerimiento, por lo que se podría asumir que la causa de la presencia de estas enfermedades responda a una muy poca actividad física. Por otro lado, el principal problema en la alimentación de este grupo fue la deficiencia en la ingesta de fibra dietaria, de grasa y de folatos.
\end{abstract}

Palabras clave: Mujeres adolescentes, sobrepeso, obesidad, requerimientos energéticos, requerimientos alimentarios.

\section{Abstract}

Introduction: Overweight and obesity result from change in dietary patterns and increasingly sedentary attitude. Objectives: To compare the energy and nutrient intake in female adolescents with overweight and obesity. Design: Cross-sectional, observational and analytical study. Location: Lima's urban area Education Center. Participants: Adolescents aged 10 to 18 years. Interventions: One hundred overweight adolescents and 87 obese adolescents were studied. Diagnosis was done by body mass index (BMI) with the World Health Organization (WHO) reference population and cutoff levels between 85 and 95 percentile for overweight and over 95 for obesity. Amount of energy and nutrients was determined by a semi quantitative food frequency questionnaire using the Peruvian Table for Food Composition and the Table of the USA Department of Agriculture. FAO recommendations were used to determine the adequate percentage of energy and the quantitative identification of vitamin and mineral intake. Statistical analysis used Student t-test to compare measures. Main outcome measures: Energy intake, ingested nutrients, vitamins and minerals adequacy according to the study groups. Results: There was no difference in energy intake between the groups studied, and these values were lower than requirements. Adequacy of fat consumption, saturated, monounsaturated and polyunsaturated fats was below requirements; however saturated fat was more consumed. The amount of vitamins and minerals ingested covered requirements except for folates. Fiber intake was very poor. Conclusions: Neither group exceeded energy requirements, so it could be assumed that cause of these conditions was very little physical activity. On the other hand the main problem was dietary fiber, fat and folate intake deficiency.

Key words: Female adolescents, overweight, obesity, energy requirements, nutrition requirements.

An Fac med. 2013;74(1):15-20 


\section{INTRODUCCIÓN}

La Organización Mundial de la Salud (OMS) ha sido muy clara en identificar que los cambios en los patrones dietarios y en la actitud cada vez más sedentaria son los elementos principales en las modificaciones que se están dando en los estilos de vida, y que esto a su vez ha devenido en la aparición de la obesidad y consecuentemente de enfermedades como la diabetes mellitus tipo 2, hipertensión arterial, dislipidemias y ciertos tipos de cánceres ${ }^{(1)}$.

En lo que se refiere a los patrones dietarios, dicho estudio analiza los cambios que se han dado, a través del tiempo, desde la época de los cazadores pasando por la de los agricultores hasta la actualidad, que es considerada como la época moderna. En todo este tiempo, los cambios de mayor trascendencia han sido la disminución importante de la ingesta de fibra y el incremento en el consumo de azúcar, sal y grasas a predomino de las saturadas, dieta conocida como 'afluente' y que conjuntamente con el sedentarismo han sido reconocidos como responsables del aumento de las prevalencias de las enfermedades mencionadas ${ }^{(1)}$.

El sobrepeso y la obesidad ya son consideradas como entidades patológicas, cuyas implicaciones están dadas en la esfera de la salud, en lo social e incluso en lo económico. El tener estas patologías implica la posibilidad de tener riesgo cardiovascular y este riesgo se hace mucho más dramático y peligroso cuando se presenta a temprana edad (2).

Diferentes estudios realizados en el país muestran una presencia importante de sobrepeso y obesidad en los niños escolares y adolescentes de clases sociales consideradas como medias y bajas ${ }^{(3)}$. Pero, lo más llamativo por su magnitud, es la prevalencia de estas enfermedades en la clase alta ${ }^{(4)}$.

La relación que existe entre la alimentación, el sobrepeso, la obesidad y sus comorbilidades ha sido muy bien demostrada en Corea, Polonia y Japón.
En Corea, en base a la comparación de tres grandes estudios (1998, 2001, 2005), en adolescentes, se ha demostrado el incremento de la prevalencia de sobrepeso y obesidad, la disminución de los patrones de alimentación tradicional y el incremento en los patrones de alimentación occidentales, que de alguna manera se refleja en el aumento de la ingesta energética y en el porcentaje de grasa, y que esto último puede afectar negativamente la salud para cuando lleguen a la adultez ${ }^{(5)}$.

En Polonia, como consecuencia de las transformaciones políticas y económicas, producidas a partir de los 80 , disminuyó la compra de alimentos como mantequilla, manteca y aceites, lo que llevó a un mayor consumo de grasas vegetales. Este cambio forzado en la alimentación se vio reflejado en una disminución de las tasas de enfermedades crónicas no transmisibles y esto respondió a que grasas poliinsaturadas desplazaron a las saturadas ${ }^{(6)}$.

En el Japón han observado que una dieta 'saludable' fue asociada con un menor riesgo de obesidad. Esta dieta se caracteriza por un elevado consumo de vegetales, hongos, algas, pescado, soya, frutas, pescados procesados. En contraste, la dieta tradicional japonesa (rica en arroz, miso y productos de soya) y las dietas occidentalizadas (ingestas altas de carne, grasas, aceites, condimentos, huevos), ambas fueron significativamente asociadas con un incremento en el riesgo de la obesidad ${ }^{(7)}$.

El objetivo del presente estudio fue comparar la ingesta de energía y nutrientes en adolescentes mujeres con sobrepeso y obesidad.

\section{MÉTODOS}

La población estuvo conformada por 1185 escolares de género femenino, entre 10 y 17 años, de un Centro Educativo ubicado en el cercado de Lima.

El estudio, en una primera etapa, pesó y talló a toda la población. Las medidas fueron hechas siguiendo las recomendaciones internacionalmente aceptadas ${ }^{(8)}$. Se determinó el índice de masa corporal (IMC) (peso/talla ${ }^{2}$ ) expresado en kilogramos por metro cuadrado $\left(\mathrm{kg} / \mathrm{m}^{2}\right)$.

La identificación del sobrepeso y obesidad fue en base a la población de referencia dada por la Organización Mundial de la Salud (OMS) ${ }^{(9)}$ y con los siguientes criterios diagnósticos, valores de 85 a 95 percentil para el sobrepeso y mayores del 95 percentil para la obesidad.

Para evaluar la ingesta alimentaria de los adolescentes se aplicó un cuestionario de frecuencia de consumo semicuantitativa. Mediante dicho instrumento se preguntó a la madre y/o tutor de la adolescente con qué frecuencia (diaria, semanal o mensual) se consumía los alimentos incluidos en una lista de 53 alimentos. Este cuestionario fue aplicado a 197 adolescentes. Se eliminó las encuestas con datos incompletos $(\mathrm{n}=10)$, siendo válidas $187 ; 100$ de ellas correspondió a las que presentaron sobrepeso y el resto a las obesas

El cálculo del contenido de energía y nutrientes, a partir de los alimentos ingeridos fue hecho utilizando la Tabla Peruana de Composición de Alimentos ${ }^{(10)}$. La Tabla de Composición de Alimentos del INCAP (11) y las Tablas del Departamento de Agricultura de los EE UU ${ }^{(12)}$ fueron usadas en el caso de aquellos nutrientes no encontrados en la Tabla Peruana.

Para el cálculo del porcentaje de adecuación de energía se utilizó como referencia las recomendaciones de la FAO (2001) ${ }^{(13)}$ y se tomó el peso ideal para cada año de edad de las encuestadas (tabla 1). En lo referente a la adecuación de proteínas, se tuvo en cuenta las recomendaciones de la FAO (2004) $^{(14)}$, utilizando el peso corporal ideal antes mencionado. La cantidad requerida es de $0,85 \mathrm{~g} / \mathrm{kg} \mathrm{PC} / \mathrm{d}$, pero se corrigió a $1 \mathrm{~g} / \mathrm{kg} \mathrm{PC} / \mathrm{d}$ por la calidad de la proteína de la dieta.

En lo que respecta al cálculo de la adecuación de minerales y vitaminas, se utilizó el documento de la FAO 
Tabla 1. Requerimiento de energía y proteínas de las adolescentes según el peso ideal.

\begin{tabular}{ccccc} 
Edad, años & Peso & $\mathrm{kcal} / \mathrm{kg} / \mathrm{día}$ & $\begin{array}{r}\text { Requerim. energía, } \\
\mathrm{kcal} / \mathrm{d}\end{array}$ & $\begin{array}{c}\text { Requerim. proteína, } \\
\mathrm{g} / \mathrm{d}\end{array}$ \\
9 & 30,5 & 60,8 & 1854 & 30,5 \\
10 & 34,7 & 57,8 & 2006 & 34,7 \\
11 & 39,2 & 54,8 & 2148 & 39,2 \\
12 & 43,8 & 52,0 & 2278 & 43,8 \\
13 & 48,3 & 49,3 & 2381 & 48,3 \\
14 & 52,1 & 47,0 & 2449 & 52,1 \\
15 & 55,0 & 45,3 & 2492 & 55,0 \\
16 & 56,4 & 44,4 & 2504 & 56,4 \\
17 & 56,7 & 44,1 & 2500 & 56,7 \\
\hline
\end{tabular}

Tabla 2. Promedio y desviación estándar de energía, macronutrientes y fibra en adolescentes con sobrepeso y obesidad.

\begin{tabular}{cccccc} 
& \multicolumn{2}{c}{ Sobrepeso, $\mathrm{n}=100$} & & \multicolumn{2}{c}{ Obesidad, $\mathrm{n}=87$} \\
\cline { 2 - 3 } \cline { 5 - 6 } Energía, kcal & Promedio & $\mathrm{DE}$ & & Promedio & $\mathrm{DE}$ \\
Proteína, $g$ & 2062 & 498 & & 2047 & 479 \\
Grasa, $\mathrm{g}$ & 79,2 & 17,2 & & 80,2 & 19,7 \\
Hidratos de carbono, $g$ & 46,9 & 14,7 & & 44,7 & 13,2 \\
Fibra, g & 339,0 & 94,1 & & 337,8 & 91,1 \\
\hline
\end{tabular}

Tabla 3. Promedio y desviación estándar de grasa animal, vegetal, total, saturada, mono insaturada, poliinsaturada y colesterol, en adolescentes con sobrepeso y obesidad.

\begin{tabular}{cccccc} 
& \multicolumn{2}{c}{ Sobrepes0, $\mathrm{n}=100$} & & \multicolumn{2}{c}{ Obesidad, $\mathrm{n}=87$} \\
\cline { 2 - 3 } \cline { 5 - 6 } & Promedio & $\mathrm{DE}$ & & Promedio & $\mathrm{DE}$ \\
Grasa total, g & 46,9 & 14,7 & & 44,7 & 13,2 \\
Grasa animal, $\mathrm{g}$ & 32,0 & 11,1 & & 30,4 & 10,3 \\
Grasa vegetal, $\mathrm{g}$ & 14,9 & 6,5 & & 14,3 & 6 \\
Grasa saturada, g & 18,2 & 6,1 & & 16,9 & 5,6 \\
G. Monoinsaturada, g & 13,4 & 4,9 & & 12,3 & 4,6 \\
G. Poliinsaturada, g & 12,6 & 4,2 & & 12,4 & 4,3 \\
Colesterol, mg & 268,5 & 98,2 & & 275,9 & 114,8 \\
\hline
\end{tabular}

Tabla 4. Promedio y desviación estándar de la ingesta de vitaminas en adolescentes, según estado nutricional.

\begin{tabular}{|c|c|c|c|c|c|}
\hline \multirow{2}{*}{ Vitaminas } & \multirow{2}{*}{ Requerimiento } & \multicolumn{2}{|c|}{ Sobrepeso } & \multicolumn{2}{|c|}{ Obesidad } \\
\hline & & Promedio & $\mathrm{DE}$ & Promedio & $\mathrm{DE}$ \\
\hline Retinol, $\mu \mathrm{g}$ & 600 & 1306,4 & 921,9 & 1243,1 & 924,1 \\
\hline Tiamina, mg & 1,1 & 1,6 & 1,1 & 1,7 & 1,2 \\
\hline Riboflavina, mg & 1,0 & 2,0 & 0,6 & 1,9 & 0,6 \\
\hline Niacina, mg & 16,0 & 17,9 & 5,0 & 18,3 & 5,8 \\
\hline Vitamina C, mg & 40,0 & 156,9 & 114,4 & 138,7 & 88,3 \\
\hline Piridoxina, mg & 1,2 & 2,2 & 0,7 & 2,2 & 0,7 \\
\hline Folatos, $\mu \mathrm{g}$ & 400,0 & 331,0 & 142,1 & 319,1 & 131,2 \\
\hline Cobalamina, $\mu \mathrm{g}$ & 2,4 & 10,3 & 7,5 & 10,1 & 7,6 \\
\hline
\end{tabular}

(2002) ${ }^{(15)}$. En el caso de minerales, como el fósforo, sodio y potasio, se tomó el DRI, de $1997^{(16)}$, para el primero y el DRI del 2005 para los dos últimos ${ }^{(17)}$.

Las encuestas fueron digitadas en una plantilla en MS EXCEL, que proporcionaba la cantidad de energía y nutrientes por raciones de alimento consumido. El análisis de datos se llevó a cabo con el programa SPSS versión 15 y son presentadas como promedio y desvío estándar. Se utilizó la prueba $t$ de Student para la significación estadística.

\section{RESULTADOS}

En la tabla 2 se observa que los que tenían sobrepeso ingirieron $2062 \mathrm{kcal}$, mientras que los obesos $2047 \mathrm{kcal}$; estas diferencias no fueron estadísticamente significativas. En lo que se refiere a los macronutrientes, que son los responsables del aporte de energía, no existió diferencia estadística; lo mismo sucedió con la ingesta de fibra dietaria.

La tabla 3 muestra que el consumo de grasa fue muy similar entre las adolescentes con sobrepeso y con obesidad. En cuanto a los orígenes de la grasa, fue mayor de las que provenían del reino animal en relación al vegetal, tanto para los con sobrepeso y obesidad. En cuanto a la composición, se ingirió más grasa saturada que mono y poliinsaturada, en ambas categorías nutricionales. La ingesta de colesterol fue más o menos parecida. Ninguna de las diferencias mencionadas fue estadísticamente significativa.

La distribución porcentual de los macronutrientes en relación al valor calórico total mostró que $64,5 \%$ de la energía provino de los hidratos de carbono, $20,2 \%$ de la grasa y $15,2 \%$ de las proteínas, en las adolescentes con sobrepeso; y $64,8 \%, 19,6$ y $15,5 \%$ en las obesas, respectivamente. Las diferencias entre los grupos no presentaron significación estadística (figura 1).

Respecto al consumo de vitaminas, se observa que las ingestas de todas las 


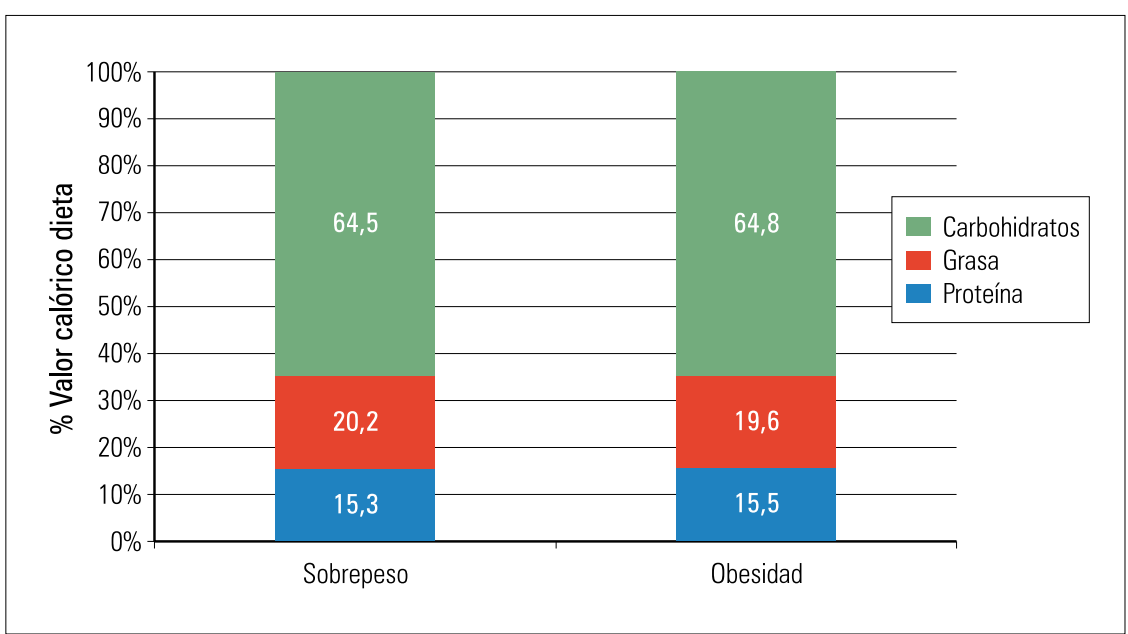

Figura 1. Distribución porcentual de la energía que aportaron los macronutrientes en adolescentes, según estado nutricional.

vitaminas, a excepción de los folatos, superaron los requerimientos, tanto en los adolescentes con sobrepeso como en los que presentaban obesidad. Las diferencias entre las ingestas en ambos grupos no fueron estadísticamente significativas (tabla 4).

En cuanto al consumo de los diferentes minerales, no se observó diferencia estadística entre las adolescentes con sobrepeso y las que tenían obesidad. A excepción del calcio, fósforo y potasio, cuyas ingestas fueron menores que el requerimiento, las ingestas de zinc y el magnesio cubrían sus requerimientos. En el caso del hierro, solo cubrieron sus requerimientos las adolescentes comprendidas entre los 10 a 14 años. El sodio fue consumido en cantidades menores a lo recomendado, pero para este caso no se ha considerado la sal (tabla 5).

\section{DISCUSIÓN}

El sobrepeso y la obesidad son el resultado de un desequilibrio energético que responde a un balance positivo de energía y esto puede deberse a que existe una mayor ingesta o un menor gasto de la misma.

En el presente estudio, y en lo que se refiere a la ingesta de energía, existen dos hechos que llaman la atención; uno es que la ingesta de las adolescentes con obesidad no presentó diferencia estadística con las que tenían sobrepeso; y el otro es que estos valores de ingesta fueron menores que los requerimientos.

Tabla 5. Promedio y desviación estándar de la ingesta de la ingesta de minerales en adolescentes con sobrepeso y obesidad, en relación a su requerimiento.

\begin{tabular}{|c|c|c|c|c|c|}
\hline \multirow{2}{*}{ Minerales } & \multirow{2}{*}{ Requerimiento } & \multicolumn{2}{|c|}{ Sobrepeso } & \multicolumn{2}{|c|}{ Obesidad } \\
\hline & & Promedio & $\overline{D E}$ & Promedio & $\mathrm{DE}$ \\
\hline Calcio, mg & 1300,0 & 638,8 & 236,5 & 597,0 & 204,7 \\
\hline Fósforo, mg & 1250,0 & 1069,1 & 281,5 & 1072,6 & 272,7 \\
\hline Hierro, mg & 12,0 & 12,5 & 3,5 & 12,6 & 3,7 \\
\hline Zinc, mg & 7,8 & 7,8 & 1,9 & 7,9 & 2,3 \\
\hline Magnesio, mg & 230,0 & 316,5 & 98,8 & 313,1 & 84,8 \\
\hline Sodio, mg & 2300,0 & 1708,8 & 729,6 & 1668,2 & 699,2 \\
\hline Potasio, mg & 4700,0 & 3307,5 & 1105,7 & 3248,2 & 947,5 \\
\hline
\end{tabular}

En el Perú se han realizado algunos estudios de consumo en el grupo de niños y adolescentes que presentan sobrepeso u obesidad; en uno se muestra mayor ingesta de energía en los obesos en relación a los que tienen sobrepeso ${ }^{(18)}$, en otro se observa lo mismo a favor de los obesos pero en relación a los normales ${ }^{(19)}$, mientras que en un tercero se encuentra una mayor ingesta de los normales frente a los obesos ${ }^{(20)}$. En este sentido, los estudios realizados no son coincidentes; pero, lo llamativo es que la ingesta de energía de los obesos no es mayor que los requerimientos de energía.

Es de esperar que exista una mayor ingesta de energía en aquellos que presentan obesidad en relación a los que tienen sobrepeso; en algunos estudios realizados en el Perú se ha dado esta premisa, pero no en todos. Estas contradicciones también han sido encontradas por Jackson, en un trabajo que realizaron en 4 poblaciones de origen africano, en las que en alguna hubo una asociación positiva entre la ingesta y la obesidad, mientras que en otra la asociación fue negativa; y esto pudiese deberse a un posible sub-reporte ${ }^{(21)}$. De la misma manera, se comunica en España que las mujeres con sobrepeso y obesidad consumen un promedio de $1606 \mathrm{kcal}$ en relación a las normales, que ingieren $2135 \mathrm{kcal}{ }^{(22)}$. Tampoco Lima y col., trabajando con un grupo de adolescentes de Brasil, encontraron diferencias en la ingesta energética de adolescentes mujeres con sobrepeso y con obesidad ${ }^{(23)}$. El estudio de DeBar comunicó que la ingesta de energía en adolescentes obesas era menor que lo recomendado para esa edad ${ }^{(24)}$.

La explicación de esta situación podría deberse a que los requerimientos de energía han sido revisados recientemente basado en los estudios hechos con el agua doblemente marcada. En función a esto, las recomendaciones actuales de la Food and Agriculture Organization of the United Nations, comparando con la anterior muestra, son entre 5 a 20\% más bajas para los niños 
y niñas menores de 12 años y 12\% más altas para los mayores de 12 años ${ }^{(25)}$.

También, habría que tener en cuenta que toda encuesta de consumo de alimentos no puede ser estimada sin considerar el error. Beaton se refería a que estos errores eran clasificados en dos grandes categorías, el sesgo y el azar, y que ambos se pueden dar tanto en el informante como en el acopiador de esa información ${ }^{(26)}$. Collins hace una revisión de estos errores y recomienda estrategias para minimizarlos ${ }^{(27)}$. Pero, la mejor recomendación, que evita por completo los errores, es usar marcadores, como por ejemplo, el agua doblemente marcada, que es un método no invasivo y permite medir el gasto energético.

En lo que concierne a la distribución porcentual de los macronutrientes de la energía ingerida, los carbohidratos y proteínas aportan un porcentaje más o menos similar a los requerimientos, lo que no sucede con la grasa. Existe una creencia popular de que la ingesta de grasa es la culpable de la obesidad y nada hay más alejado de la realidad. En una dieta equilibrada se encuentra cubriendo el 30\% de la energía que se consume y, por lo que se puede apreciar en el presente estudio, la grasa solo alcanza al 20\%, tanto en las adolescentes con sobrepeso como en las obesas. En ninguno de los estudios antes mencionados, el consumo de grasa llega al $30 \%{ }^{(18-20)}$. Lo que sí sería preocupante, y donde estos estudios llegan a la misma conclusión, es que esa grasa es a predominio de la saturada, en función de la mono y poliinsaturada. Sin embargo, es menester tomar en cuenta que estos tipos de grasa deben guardar cierta armonía entre ellas o sea cada una participa con un $10 \%$ dentro de toda la energía ingerida. En este estudio, ninguna de ellas cumplió con esa recomendación, pero la que predominó fue la saturada. En cuanto al colesterol, su ingesta estuvo por debajo de lo recomendado (300 mg). Estudios realizados en México también encuentran en adolescentes obesos una ingesta menor al 30\% de grasas ${ }^{(28)}$.
La única característica, encontrada en nuestro estudio, en común a las dietas 'afluentes' es la ingesta pobre de fibra dietaria. Otros estudios también informan lo mismo ${ }^{(18-20)}$. La fibra dietaria se encuentra en la cascarilla de los cereales, menestras, alimentos integrales; pero, principalmente en las verduras y frutas. Estas últimas, por su composición química, no solo aportan fibra sino también flavonoides, vitaminas antioxidantes, y representan un gran aporte a la buena salud; por lo contrario, una deficiencia de las mismas ha sido relacionada con la enfermedad.

Yoo encontró que el consumo bajo de estos alimentos acompañado de un consumo alto de bebidas azucaradas está independientemente asociado con la prevalencia de síndrome metabólico (SM) ${ }^{(29)}$. Así mismo, una dieta rica en frutas y verduras y baja en grasas saturadas protege de una serie de enfermedades cardiovasculares y de ciertos cánceres ${ }^{(30)}$. En un estudio realizado en 20069 personas durante 10 años, se determinó que el consumo de frutas y hortalizas disminuyó la incidencia de enfermedades cardiovasculares ${ }^{(31)}$. Esto puede deberse a un efecto positivo sobre la función endotelial ${ }^{(32)}$.

Pero no solo se recomienda cantidad, sino fundamentalmente variedad, dado que así se puede asegurar que todas las substancias de cada alimento se potencialicen ${ }^{(33)}$. En Puerto Rico, se ha encontrado una asociación entre la mayor variedad en la ingesta de frutas y verduras con una disminución de la proteína-C-reactiva (PCR), que es un indicador inflamatorio; y que, por este mecanismo, se observa la respuesta a una mayor ingesta de antioxidantes ${ }^{(34)}$. En otro estudio llevado a cabo en ancianos, se observó que un mayor consumo de frutas y hortalizas se asoció a niveles bajos de PCR y homocisteína ${ }^{(35)}$. Togo ha comunicado que dietas ricas en verduras y frutas son asociadas negativamente con altos IMC ${ }^{(36)}$.

La validación del reporte de los informantes sobre el consumo debería ser refrendada con marcadores; para el caso de las frutas y verduras, lo mejor es dosar carotenoides del plasma. En ese sentido, los trabajos de Burrow y col. mostraron que el consumo de la ingesta de frutas y verduras convertida en carotenos (alfa, beta, criptoxantina y luteína) tiene ligeras diferencias entre niños normales, con sobrepeso y obesos, pero que no son estadísticamente significativas; lo que sí muestra significación estadística son los valores de caroteno en el plasma, que van disminuyendo conforme se incrementa el IMC ${ }^{(37)}$.

En lo que respecta al azúcar, lamentablemente es el alimento más consumido, seguido por la leche y los cereales; entre ellos, el pan y el arroz fueron los preferidos. Esta preferencia por el azúcar refleja de alguna manera la mala costumbre de empezar a agregarlo en el biberón, lo que crea un hábito considerado nocivo.

El otro elemento de la dieta 'afluente' es la sal, vehículo del sodio, que cuando se consume en grandes cantidades es negativo para la salud. Además, se ingiere sodio con los alimentos. En este estudio se ha evaluado solo lo que se refiere al alimento y el consumo se encontró por debajo de la recomendación. Esto de ninguna manera nos garantiza que el consumo del sodio sea lo conveniente. Sin embargo, la OMS ha recomendado que el consumo de sal disminuya a 5 gramos por persona y día, con la finalidad de prevenir problemas cardíacos y/o renales ${ }^{(38)}$.

Dentro de las vitaminas estudiadas, las diferencias encontradas entre los grupos no fueron estadísticamente significativas. Todas, a excepción de los folatos, son ingeridas en cantidades que cubren los requerimientos. Dos situaciones preocupantes genera esta deficiencia; una es referente a que la población estudiada es del género femenino y en edad fértil, debiendo mejorar su ingesta para un posible embarazo y así prevenir un posible defecto en el tubo neural; y el otro aspecto es el que tiene que ver con su relación con la homocisteína, ya que esta deficiencia, conjuntamente con la de B12, podría ser 
causa de una hiperhomocisteinemia. El consumo de folatos y de cianocobalamina fue más alto que los promedios encontrados en una sub-muestra de escolares de 10 a 19 años con sobrepeso y obesidad (135,6 $\mu \mathrm{g}$ de folatos y 3,3 $\mu \mathrm{g}$ de cianocobalamina) ${ }^{(39)}$.

En conclusión, se puede afirmar que tanto el sobrepeso como la obesidad no responden a un desequilibrio energético, o sea a un mayor consumo de energía, por lo que se podría asumir que la causa de la presencia de estas enfermedades responda a una muy poca actividad física. Por otro lado, el principal problema en la alimentación de este grupo fue la deficiencia en la ingesta de fibra dietaria.

\section{REFERENCIAS BIBLIOGRÁFICAS}

1. Organización Mundial de la Salud (OMS). Dieta nutrición y prevención de enfermedades crónicas. Serie de Informes Técnicos 797. Ginebra 1990.

2. Pajuelo J, Bernui I, Rocca J, Torres L, Soto L. Marcadores bioquímicos de riesgo cardiovascular en una población adolescente femenina con sobrepeso y obesidad. An Fac med. 2009;70(1):7-10.

3. Pajuelo J. La obesidad infantil en el Perú. Facultad de Medicina UNMSM. Lima, 2003.

4. Pajuelo J. La situación nutricional en niños y adolescentes de un nivel socio económico alto en Lima Metropolitana. Facultad de Medicina UNMSM. Nestlé Perú. Lima, 2008

5. Song $Y$, Park $M$, Paik $H$, Joung $H$. Secular trends in dietary patterns and obesity-related risk factors in Korean adolescents aged 10-19 years. Int J Obes. 2010;34:48-56.

6. Zatenski W, Willer W. Changes in dietary fat and declining coronary heart disease in Poland Population study. BMJ. 2005;331:187-8.

7. Okubo H, Sasaki S, Murakami K, Kim M, Takahash Y, Hosoi Y, Itabashi M, and the Freshmen in Dietetic Courses Study II group. Three major dietary patterns are all independently related to the risk of obesity among 3760 Japanese women aged 18-20 years. Int J Obes. 2008;32:541-9.

8. Lohman T, Roche A. Anthropometric Standardization Reference Manual. Illinois Champaing: Human Kinetic Books. 1990.

9. Organización Mundial de la Salud (OMS). Patrones de Crecimiento Infantil de la OMS. Departamento de Nutrición para la Salud y el Desarrollo. 2006.

10. Collazos C, Alvistur J, Enrique GJ, Quiroz A, Herrera N, et al. La composición de los alimentos peruanos. $6^{\text {a }}$ ed. Lima: Ministerio de Salud-Instituto Nacional de Salud-Instituto de Nutrición, 1996.

11. Menchú MT, Mendez H (eds.). Tabla de composición de Alimentos de Centroamérica. $2^{\circ}$ edición. Guatemala: INCAP/OPS, 2006.

12. United States Department of Agriculture. Agricultural Research Service. Nutrient Data. Disponible en: http://www.ars.usda.gov/nutrientdata

13. FAO. Human energy requirements. Report of a joint FAO/WHO/UNU Expert Consultation. Rome. 2001.

14. World Health Organization and Food and Agriculture Organization of the United Nations. Vitamin and Mineral Requeriments in Human Nutrition. Second Edition. 2004.

15. FAO/WHO. Human vitamin and mineral requirements. Report of a joint FAO/WHO expert consultation. Bangkok. Thailand. Update of march 12, 2002.

16. Institute of Medicine. Food and Nutrition Board. Dietary Reference Intakes for Calcium, Phosphorus, Magnesium, Vitamin D, and Fluoride. 1997.

17. Institute of Medicine. Food and Nutrition Board. Dietary Reference Intakes for Water, Potassium, Sodium, Chloride, and Sulfate. 2005. Disponible en: http://books.nap.edu/catalog/10925.html

18. Álvarez T. Relación entre factores de riesgo cardiovascular y la ingesta de energía y nutrientes en adolescentes con sobrepeso $u$ obesidad de la Institución Educativa Scipión Llona, Miraflores. Tesis para optar el Título Profesional de Licenciada en Nutrición. Escuela Académico Profesional de Nutrición. Facultad de Medicina. UNMSM. Lima 2008

19. Pajuelo J, Bernui I, Quiroz G, Quispe J. Características alimentarias y horas de ver TV en niñas de 6 años, normales y obesas. Consensus. 2005;9(10):9-16

20. Liria M, Mispireta M, Lanata C, Creed-Kanashiro H. Perfil Nutricional en Escolares de Lima y Callao. Instituto de Investigación Nutricional. ISIL. 2008.

21. Jackson M, Walker S, Crickshank J, Sharma S, Cade J, Mbanya J, Younger N, Forrester T, Wilks R. Diet and overweight and obesity in populations of African origin: Cameroon, Jamaica and the UK. Public Health Nutrition. 2006;19(2):122-39.

22. Ortega Anta R, Carvajal A, Requejo Marcos A, López A, et al. Hábitos alimentarios e ingresos de energía y nutrientes en adolescentes con sobrepeso en comparación con los de peso normal. An Esp Pediatr. 1996;44:203-8.

23. Lima S, Arrais Pedroza L. Avaliacao de dieta habitual de criancas e adolescents com sobrepesoe obesidades. Rev Nutr Campinas. 2004;17(4):469-77.

24. De Bar L, Stevens V, Perrin N, Wu P, Pearson S, et al. A primary cared-based, multicomponent lifestyle intervention for overweight adolescents females. Pediatric. 2012;129:e611-e620.

25. Food and Agricultural Organization (FAO). Human Energy Requirements. Report of a Joint Expert Consultation. Food and Agricultural Organization: Rome, 2004.
26. Beaton G. Approaches to analysis of dietary: relationship between planned analysis and choice of methodology. Am J Clin Nutr. 1994;59:253S-261S.

27. Collins C, Watson J, Burrows T. Measuring dietary intake in children and adolescents in the context of overweight and obesity. Int J Obes. 2009;1-13.

28. Romero Velarde E, Campollo Rivas O, Castro Hernandez J, Cruz Osorio R, Vasquez Garibaldi E. Hábitos de alimentación e ingestión de calorias en un grupo de niños y adolescentes obesos. Bol Med Hosp Infant México. 2006;63(3):145-50.

29. Yoo S, Nichlas T, Baranowski T, Zakeri I, Yang S, Srinivasan R, Berenson G. Comparison of dietary intakes associated with metabolic syndrome risk factors in young adults: the Bogalusa Heart Study. Am J Clin Nutr. 2004;80:841-8.

30. Joffe M, Robertson A. The potential contribution of increased vegetable and fruit consumption to health gain in the European Union. Public Health Nutrition. 2004;4:893-901.

31. Oude Griep LM, Geleijnse JM, Kromhout D, Ocké MC, Verschuren WM. Raw and processed fruit and vegetable consumption and 10-year coronary heart disease incidence in a populationbased cohort study in the Netherlands. PLoS One. 2010;5(10):e13609.

32. Cuevas A, Germain A. Diet and endothelial function. Biol Res. 2004;37:225-30.

33. US Department of Health and Human Services. US Department of Agriculture. $6^{\text {th }}$ ed. Washington Department DC. 2005.

34. Bhupathiraju S, Tucker K. Greater variety in fruit and vegetable intake is associated with lower inflammation in Puerto Rico adults. Am J Clin Nut. 2011;93:37-46.

35. Gao X, Bermudez OI, Tucker KL. Plasma-Creactive protein and homocysteine concentrations are related to frequent fruit and vegetables intake in Hispanic and non-Hispanic white elders. J Nutr. 2004;134:913-8.

36. Togo P, Osler M, Sorensen T, Heitmann B. Food intake patterns and body mass index in observational studies. Int J Obes Relat Metab Disord. 2001;25:1741-51.

37. Burrow TL, Warren JM, Colyvas K, Garg ML, Collins CE. Validation of overweight children's fruit and vegetables intake using plasma carotenoids. Obesity. 2009;17(1):162-8.

38. Organización Mundial de la Salud (OMS). Dieta, nutrición y prevención de enfermedades crónicas. Serie de Informes Técnicos 916. Ginebra 2003.

39. Pajuelo J, Bernui I, Nolberto V. Homocisteína, vitamina B12 y ácido fólico en niños con sobrepeso y obesidad. Diagnóstico. 2006;45(3):110-4.

Artículo recibido el 9 de julio de 2012 y aceptado para publicación el 18 de octubre de 2012.

\section{Correspondencia:}

Dr. Jaime Pajuelo Ramírez

Correo electrónico: japara18@yahoo.com 